\title{
CONOCIMIENTO DEL INGLES EN ALUMNOS INGRESANTES A LA UNIVERSIDAD NACIONAL JORGE BASADRE GROHMANN DE TACNA EN LA ESPECIALIDAD DE IDIOMA EXTRANJERO
}

\author{
Knowledge of english in students entering Jorge Basadre Grohmann \\ National University of Tacna specialty foreign language
}

\author{
Silvia Milagritos Bazán Velásquez ${ }^{1}$, Cecilia Mendoza Gómez ${ }^{2}$
}

\begin{abstract}
RESUMEN
El iniciar estudios universitarios implica que los alumnos ingresantes tengan un nivel de conocimientos que les permita entender, incrementar, adquirir y desarrollar nuevos conocimientos en un idioma extranjero, específicamente los alumnos de la especialidad de idioma extranjero, traductor e interprete del idioma inglés. Asi esta investigación se ha desarrollado con el objetivo principal de conocer a ciencia cierta cuál es el nivel real de conocimiento del idioma inglés que poseen los ingresantes en la especialidad de Idioma Extranjero, Traductor e Intérprete y de esta manera sugerir medidas correctivas que lleven a dar visos de solución al álgido problema que afecta al alumnado de esta carrera.
\end{abstract}

Palabras clave: conocimiento, ingresante, nivel, postulante.

\section{ABSTRACT}

Begining college studies implies that freshmen come with a level of knowledge that will let them understand, improve, acquire and develop new knowledge in a foreign language, specially by the students of Foreign Language Translator and Interpreter college career. So this research has been developed with the main objective of knowing for sure what the real level of English language knowledge is possessed by entrants to the specialty of Foreign Language, Translator and Interpreter and thus suggest corrective measures leading to give overtones of solution to the critical problem affecting these students.

Keywords: Knowledge, freshman, level, applicant.

\section{INTRODUCCIÓN}

Saber cuál es el nivel de conocimiento que tienen los alumnos al iniciar una carrera universitaria es de gran importancia porque en base a ello se establecerán los nuevos conocimientos que irán adquiriendo de acuerdo al tema o contenido a tratar, la concepción constructivista del aprendizaje se sustenta en la idea de que la finalidad de la educación que se imparte es promover los procesos de crecimiento personal del alumno en el marco de la cultura del grupo al que pertenece. Bajo esta perspectiva, el aprendizaje ocurre sólo si se

\footnotetext{
${ }^{1}$ Mgr. en Docencia Universitaria y Gestión Educativa, Licenciada en Educación en Idioma Extranjero. Correoelectrónico: georgettesbo@hotmail.com

${ }^{2}$ Licenciada en Educación. Docente de la Facultad de Ciencias de la Educación, Ciencias de la Comunicación y Humanidades de la Universidad Nacional de Jorge Basadre Grohmann. Tacna-Perú.
} 
satisfacen una serie de condiciones: que el alumno sea capaz de relacionar de manera no arbitraria y sustancial la nueva información con los conocimientos, experiencias previas y familiares que posee en su estructura de conocimientos, que tiene la disposición de aprender significativamente y que los materiales o contenidos de aprendizaje tienen significado potencial o lógico.

Por lo tanto, en el ámbito educativo debe tenerse en cuenta que si los alumnos tienen procesos individuales y esquemas de pensamiento previos, los docentes deben promover ambientes de aprendizaje donde las actividades de exploración, reto y descubrimiento para el alumno sean más importantes que la enseñanza en sí. De esta manera, el estudiante se convierte en el protagonista del aprendizaje y no el docente.

Así, resulta apropiado tener en cuenta los conocimientos iniciales de los estudiantes y establecer una relación coherente entre lo que los alumnos saben y los nuevos conocimientos. En caso contrario, se generarían dificultades innecesarias y falta de confianza de los alumnos para afrontar el nuevo conocimiento.

Sin embargo, no se trata de indagar exactamente todo lo que un alumno sabe, bastante inviable, sino de determinar los conocimientos iniciales de los alumnos para un nuevo aprendizaje y evitar suposiciones. Por ejemplo, en el caso de los estudiantes que ingresan en las carreras de ingenierías, pensar que ya vienen fortalecidos en los conocimientos matemáticos básicos y esto será suficiente para llevar esta carrera de manera adecuada y no aplicar instrumentos adecuados de evaluación inicial como es un examen de ingreso donde no se tome absolutamente nada de matemáticas seria trabajar sobre supuestos que no llevaría a un buen fin. En el caso de los alumnos que se presentan a la universidad para estudiar la carrera de idioma extranjero no se les toma ningún examen de conocimiento del idioma inglés. No se toma en cuenta que en los colegios nacionales solo estudian inglés en el nivel secundario con escasas dos horas pedagógicas a la semana sin darle la importancia necesaria.

Así como los alumnos postulantes a diferentes carreras dan exámenes por canales: ciencias y humanidades, a los alumnos postulantes a la carrera de idioma extranjero se les debería exigir y aplicar un examen de conocimiento previo del idioma extranjero y habilidad para aprendizajes de idiomas.

Los docentes universitarios constatamos las dificultades que presenta el alumnado para conseguir los objetivos de aprendizaje que se pretenden en los estudios superiores. Esta problemática surge constantemente en los debates entre los docentes, que esperan de los alumnos no sólo una base de conocimientos, sino también una formación básica en habilidades de aprendizaje que le permita cursar con éxito los estudios universitarios.

El estudiante, cuando finaliza su enseñanza secundaria y consigue una plaza en una facultad o escuela universitaria, tiene que haber adquirido una serie de conocimientos generales (conceptos, procedimientos y actitudes). Además, en función de los estudios que quiera cursar, tiene que haber adquirido un cierto nivel y determinadas disciplinas. Es obvio que hay un grupo de estudiantes más preparado que otro para obtener un rendimiento académico óptimo en los estudios universitarios. El estudiante debe acceder a los estudios superiores con un nivel adecuado de conocimientos previos que le permita "aprender" y cursar con éxito los estudios escogidos. 
En nuestro país los alumnos que postulan para estudiar la carrera de profesores en Idioma Extranjero no rinden un examen sobre conocimientos previos del idioma. Siendo así que muchos alumnos postulan e ingresan a esta carrera solo con el conocimiento que traen de los colegios secundarios. Donde los alumnos solo llevan el idioma inglés en una cantidad de dos horas pedagógicas y el nivel de exigencia es muy bajo limitándose solo a adquirir estructuras muy básicas y vocabulario demasiado básico.

Muchos alumnos ingresan con la idea de adquirir el idioma en la universidad, concepción totalmente equivocada ya que ellos ingresan a adquirir y desarrollar conocimientos que los llevarán a formarse como futuros docentes, traductores e intérpretes. El choque es muy grande al llevar cursos totalmente en idioma ingles ya que ellos no poseen las herramientas necesarias para poder desenvolverse de manera adecuada.

Por lo tanto es urgente no solo estudiar sino también proponer medidas correctivas y soluciones a este grave problema.

\section{Lengua extranjera}

Una lengua extranjera es una diferente de la lengua materna de una persona. La persona solamente adquiere esa lengua extranjera si la aprende de manera consciente, ya sea en una escuela, con cursos de idiomas o de manera autodidacta, o bien en el caso de los niños, cuando el aprendizaje ocurre jugando en un ámbito de lengua extranjera.

\section{Aprendizaje de idiomas extranjeros}

La edad de inicio en el aprendizaje de idiomas es determinante para obtener buenos resultados a largo plazo en el desarrollo de las habilidades comunicativas. Por tanto, cuanto antes se inicie la exposición al aprendizaje, mejor.

\section{Aprendizaje significativo en lenguas extranjeras}

La clave del aprendizaje significativo está en la relación que se pueda establecer entre el nuevo material y las ideas ya existentes en la estructura cognitiva del sujeto. Por lo expuesto, la eficacia de este tipo de aprendizaje radica en su significatividad y no en técnicas memorísticas.

Por lo tanto sino existe un adecuado nivel de conocimiento previo no se puede formar un andamiaje que pueda llevar a alcanzar un aprendizaje significativo.

Aquí cabe señalar que si el alumno llega con un conocimiento muy bajo o casi nulo esto no ayuda a alcanzar el aprendizaje significativo. Todo lo contrario esto implica empezar a construir un andamiaje que debió ser adquirido hace muchos años. Este problema se agudiza si se habla de estudiantes de nivel superior específicamente de nivel universitario.

\section{CHOQUE CULTURAL}

Este término, conocido también con el vocablo inglés cultural shock, se refiere al conjunto de reacciones que puede llegar a experimentar un individuo, y por extensión el estudiante de segundas lenguas o lenguas extranjeras, al entrar en contacto por primera vez con una cultura diferente de la propia, cuyo grado de conocimiento puede ser muy diverso entre individuos. Tal impacto suele tener una variable afectiva y una variable cognitiva. Desde el punto de vista afectivo, es posible que el sujeto experimente emociones como el miedo, la desconfianza, la incomodidad, la ansiedad o la inseguridad. Desde el cognitivo, puede experimentar un conflicto cognitivo entre su conocimiento del 
mundo por un lado y los marcos de conocimiento, los valores o las interpretaciones propias de la nueva cultura, por otro.

Algunos autores han definido el choque cultural como la pérdida de la operatividad de las estrategias de resolución de problemas que el individuo ha desarrollado en su lengua primera, cuando trata de aplicarlas al contexto de la nueva cultura. Otros han descrito el fenómeno de la aculturación, compuesto de las siguientes etapas: 1) euforia, 2) choque cultural, 3) stress cultural y 4) asimilación o aceptación.

Las investigaciones actuales se sostienen generalmente que cuanto mayor es la distancia entre la cultura propia y la extranjera mayor es el choque cultural. Sin embargo, la reacción del individuo viene condicionada por los factores personales (como, por ejemplo, la personalidad, la actitud, los conocimientos previos, la experiencia en situaciones de comunicación intercultural o las expectativas creadas en torno a la cultura en cuestión) y los factores ambientales. Parece obvio que el desarrollo de un determinado nivel de competencia comunicativa y competencia intercultural se corresponde con una mayor habilidad para superar las posibles consecuencias negativas o traumáticas de esta experiencia.

\section{El marco europeo de referencia}

El marco europeo de referencia promueve un cambio en las prácticas de enseñanza con miras a mejorar las dinámicas de las relaciones de idiomas en Europa, en donde el inglés tiene un lugar dominante. Con ello, se espera lograr la integración y aumentar la movilidad de todos los ciudadanos de la Unión Europea por medio de la competencia lingüística. El marco común de referencia europeo es flexible y abierto, basado en un espíritu de no imposición y libertad de uso; puede adaptarse a las necesidades y situaciones específicas de maestros y alumnos, a los cuales considera independientes, capaces de tomar sus propias decisiones, críticos y dotados de habilidades. Además, el marco europeo considera el idioma como un "fenómeno sociocultural, en continua evolución para responder a las exigencias de su uso en comunicación", y no solo "un aspecto principal de la cultura, sino un medio para acceder a manifestaciones culturales".

Este marco común de referencia europeo es el que define la pauta a nivel mundial en cuanto al aprendizaje, lineamientos para establecer sistemas de validación de competencias generales individuales, tales como saber, saber hacer, saber ser y saber aprender y habilidades lingüísticas, lo cual lo ha convertido en el punto de referencia para el aprendizaje, enseñanza y evaluación de idiomas aceptado en todo el mundo.

\section{Población y métodos}

Se tomó como población a estudiantes que son ingresantes en la especialidad de Idioma Extranjero, Traductor e Intérprete y se trabajó con los mismos. Ver figura 1.

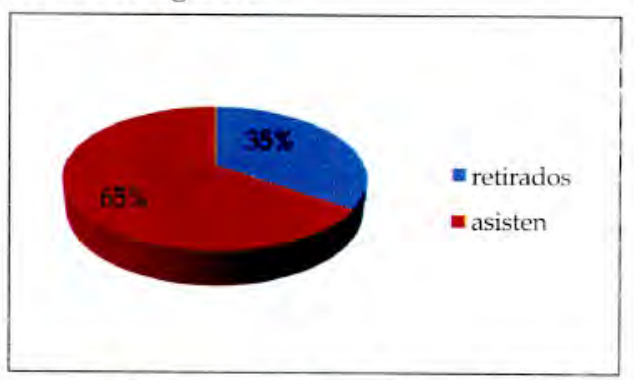

Figura 1. Ingresantes a la especialidad IETI en el año 2013.

El método utilizado para la recolección de datos fue un test de conocimiento. Como instrumento de investigación se usó la escala de estimación. 


\section{RESULTADOS}

Los resultados muestran lo siguiente:

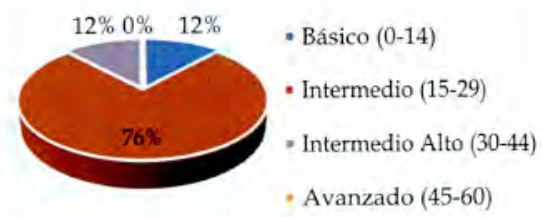

Figura 2. Puntajes obtenidos en el test de conocimiento

Tabla 1. Nivel de conocimiento de ingresantes a idioma extranjero

\begin{tabular}{lcc}
\hline Nivel & Numero & Porcentajes \\
\hline Básico (0-14) & 3 & 0,12 \\
Intermedio (15-29) & 19 & 0,76 \\
Intermedio Alto (30-44) & 3 & 0,12 \\
Avanzado (45-60) & 0 & 0 \\
& 25 & \\
\hline
\end{tabular}

1. Un $12 \%$ de alumnos se encuentran en el nivel básico del idioma.

2. Un $76 \%$ demuestran un nivel de conocimiento del idioma intermedio bajo.

3. Un $12 \%$ demuestran un nivel de conocimiento del idioma intermedio alto.

4. Ningún alumno demuestra un conocimiento del idioma en el nivel avanzado

Los resultados demuestran que los ingresantes tienen un nivel bajo y en algún caso casi nulo de conocimiento del idioma, que es insuficiente para desarrollar una carrera universitaria

La especialidad de idioma extranjero es la más numerosa entre las diferentes carreras que se ofrecen en la Facultad de Educación, sin embargo, como se observa en la figura 1 , el número de retirados es alto de $(35 \%)$ debido a diversos factores. Muchos alumnos no cuentan con el conocimiento, actitud o habilidad necesaria para seguir esta carrera, otros ingresaron en la especialidad como segunda opción esperando poder posteriormente trasladarse a otra carrera, sin embargo, el grado de exigencia de los estudios y la falta de conocimiento muchas veces básico del idioma, así como la falta de aptitud y habilidad para el aprendizaje de idiomas extranjeros no les permitió alcanzar el nivel y los requerimientos necesarios para poder lograr el objetivo de pasarse a otra carrera.

Los alumnos que asisten regularmente suman 32, esto es, los alumnos que se proyectan a terminar la carrera.

Es importante que los interesados en seguir la carrera de idioma extranjero revisen los planes de estudio de la carrera universitaria que desean estudiar minuciosamente para saber si cuentan con conocimiento, habilidades y sus aspiraciones como profesionales. Asimismo, buscar opiniones de profesionales de la carrera, orientadores vocacionales o en internet para disuadir sus dudas sobre alguna asignatura concreta.

Con respecto a la parte académi$\mathrm{ca}$, muchas veces los estudiantes no conocen tampoco el alcance de la carrera que han elegido. Piensan que se trata de una mera academia de idiomas a la cual acuden a tomar clases, de la misma forma que lo harían en un instituto. Ellos deberán tomar conciencia de que se trata de un estudio mucho más profundo y minucioso. Serán, en un futuro, profesionales de la lengua inglesa ya sea en el campo de la traducción como así también en el de la enseñanza. Los estudiantes al no contar con el nivel de inglés requerido para desempeñarse tanto en el lenguaje oral como en el escrito ceden al temor y esto da lugar a las inhibiciones, que se producen ante el temor al fracaso o a exponerse ante las críticas ajenas. Son frecuentes las inhibiciones del aprendizaje ante situaciones nuevas vivenciadas como muy amenazantes.

Los resultados arrojan que la 
mayoría de alumnos ingresantes poseen bajo nivel de conocimiento del idioma inglés y que existe un gran número de alumnos que se retiran debido a que no poseen la herramienta necesaria (conocimiento del idioma) para poder estudiar esta carrera universitaria.

\section{CONCLUSIONES}

La mayoría de los ingresantes en la especialidad de Idioma Extranjero, Traductor e Intérprete de la Universidad Nacional Jorge Basadre Grohmann no muestran un nivel adecuado para estudiar una carrera de idiomas lo que conlleva a una serie de frustraciones y deficiencias en la formación académica profesional.

Este es un problema álgido que debe ser solucionado para dar la debida formación profesional.

El nivel de conocimientos previos del idioma inglés en los ingresantes en la especialidad de Idioma Extranjero Traductor e Intérprete es bajo y esto se debe a la falta de exigencia de la universidad de un examen de conocimientos en inglés, por tanto es necesario y obligatorio que los postulantes, cuenten con conocimientos previos en el idioma, para tener una base sólida en su formación profesional y consolidarse como profesionales competentes.

Es aceptado y reconocido que para aprender un idioma extranjero mientras más temprano se aprenda es mejor. Esto quiere decir que es mejor empezar el aprendizaje en la niñez. Cuando se trabaja con alumnos adolescentes que van a formarse profesionalmente y al mismo tiempo aprender un idioma que es el caso de los ingresantes en la carrera de Idioma Extranjero Traductor e Intérprete se enfrentan a un gran reto, lo que requiere el doble de esfuerzo y dedicación de una persona para formarse en una universidad. Esto lleva en muchos casos a frustración, pésimo rendimiento, falta de aprendizaje significativo y deserción de una buena cantidad de alumnos.

Los conceptos de conocimiento previo y su importancia en el aprendizaje de un idioma extranjero que conlleva también el aprendizaje de una nueva cultura corroboran los resultados de la presente investigación.

El aprendizaje significativo por parte de los ingresantes en la carrera de Idioma Extranjero Traductor e Intérprete requiere también conocimientos previos mínimos para estudiar una carrera universitaria, al no contar con esto en muchos casos no se logra un aprendizaje significativo.

En la actualidad existe un alto grado de exigencia en el campo de formación profesional y la actividad profesional. Así como en el campo profesional. Las instituciones de formación académica son valoradas por el nivel de exigencia para el ingreso y en el nivel académico y profesional.

El mercado exige profesionales competentes y quienes no cumplen con este requisito tienen muchas dificultades para posicionarse en este mundo laboral cada vez más exigente.

Se debe incidir en que el alumno que postula a la carrera debe demostrar un adecuado nivel de conocimientos del idioma inglés que le permita una correcta formación profesional.

Al elevar el nivel de exigencia mejorara la formación de los alumnos y futuros profesionales y se podrá situarlos al mismo nivel de competitividad de otras universidades

El nivel de conocimientos previos en el idioma inglés por parte de los ingresantes en la especialidad de Idioma Extranjero, Traductor e Intérprete es muy bajo para seguir una carrera univer- 
sitaria.

Los alumnos ingresantes en la especialidad de Idioma Extranjero, Traductor e Intérprete poseen un bajo nivel de conocimiento del idioma debido a la falta de exigencia para postular e ingresar a esta carrera, y al hecho de que no rinden ninguna prueba de conocimiento del idioma inglés al postular.

Se concluye que el nivel de los estudiantes y futuros profesionales mejorará cuando la universidad implemente políticas para medir el conocimiento del postulante a la carrera de Idioma Extranjero, Traductor e Intérprete.

\section{REFERENCIAS BIBLIOGRÁFICAS}

Brinton, D., Snow, M. y Wesche, M. (1989). Content based second language instruction. Boston: Heinle \& Heinle

Carson, J., Taylor, J. y Fredella, L. (1997). The role of content in task based instruction: What is the relationship? In M. Snow y D. Brinton (eds) The content based classroom: perspectives on integrating language and content (pp. 367370). White Plains: Addison Wesley-Longman.

Chamot, A. y O'Malley, J. (1987). The Cognitive academic language learning approach. TESOL Quarterly, 21, 227-247

Ellis, R. (1992). The classroom context: an acquisition/rich or an acquisition/poor environment. In C. Kramsch y S. McConnell-Ginet (Eds) Text and context: cross disciplinary perspectives on language study (pp. 171-186). Lexington, D.C. Heath.

Falk, J.S. (1978). Linguistics and language, EE. UU.: John Wiley \& Sons.

Fasold, R.W. and Connor-Linton J.
(2006). An introduction to language and linguistics, Cambridge: Cambridge University Press.

Hollmann, J. A. (2010). Language \& the brain: The neural basis of foreign language proficiency. Hamburgo: Verlag Dr. Kovac.

Hymes, D. (1971). On communicative competence. Philadelphia: University of Pennsylvania Press

Krashen, S. (1985). The input hypothesis: issues and implications. New York: Longman

Krashen, S. (1981). Second language acquisition and second language learning. New York: Prentice Hall.

Steinberg, D. D. (1991). Psycholinguistics: language, mind and world. Londres: Longman.

Stern, H. H. (1983). Fundamental concepts of language teaching. Oxford: Oxford University Press.

Pally, M. (2000). Sustained content teaching in academic ESL/EFL. Boston: Houghton Mifflin Company.

Richards, J.C. y Schmidt R. (2002). Longman dictionary of language teaching and applied linguistics, $3^{\mathrm{a}}$ ed. Londres: Longman

Ponder, R. Y Powell, W. (1989). Source books as content bearing instruction in intensive ESL programs. Citado en Pally (2000)

Prabhu, N. (1987). Second language pedagogy. Oxford, OUP.

Swain, M. (1985). Communicative competence: some roles of comprehensible input and comprehensible output in its development. In S. Gass y C. Madden.

Widdowson, H. (1990). Aspects of languageteaching. Oxford, OUP

Widdowson, H. (1993). The relevant conditions of language use and learning. 